\title{
Einsteinian gravity from a topological action
}

\author{
Eckehard W. Mielke ${ }^{1}$ \\ Universidad Autónoma Metropolitana-Iztapalapa, \\ Apartado Postal 55-534, C.P. 09340, México, D.F., MEXICO
}

\begin{abstract}
The curvature-squared model of gravity, in the affine form proposed by Weyl and Yang, is deduced from a topological action in $4 \mathrm{D}$. More specifically, we start from the Pontrjagin (or Euler) invariant. Using the BRST antifield formalism with a double duality gauge fixing, we obtain a consistent quantization in spaces of double dual curvature as classical instanton type background.

However, exact vacuum solutions with double duality properties exhibit a 'vacuum degeneracy'. By modifying the duality via a scale breaking term, we demonstrate that only Einstein's equations with an induced cosmological constant emerge for the topology of the macroscopic background. This may have repercussions on the problem of 'dark energy' as well as 'dark matter' modeled by a torsion induced quintaxion.
\end{abstract}

Keywords: Topological gravity, double duality, BRST quantization, Einsteinian background PACS: 04.20.Fy, 04.60-m, 04.50.+h, 11.15-q

\section{INTRODUCTION}

On the macroscopical level, Einstein's general relativity has passed every test "with flying colors", see Will [67] for a recent review. However, Einstein's theory sofar has resisted any attempt of quantization, e.g. it is known to be perturbatively nonrenormalizable, partially due to its dimensional coupling constant ${ }^{2}$. String theory or brane scenarios with extra dimensions have been proposed as a rescue, some of which are implying, however, deviations of standard gravity in the sub-millimeter range. Recent torsion balance experiments [28] have probed the inverse square law and found no deviation even below the hypothetical dark energy (DE) scale of $\lambda_{\mathrm{DE}}=\left(\hbar c / \rho_{\mathrm{DE}}\right)^{1 / 4} \simeq$ $85 \mu \mathrm{m}$. Thus this 'window' of possibly new gravitational physics seems also to be closing.

In 1974 Yang proposed an affine gauge theory gravity [70] which, due to its scale invariance, can be regarded as a rather promising fundamental theory of (quantum) gravity in the high-energy limit [25], without invoking extra dimensions or supersymmetry, cf. Ref. [30]. An additional duality constraint on the curvature could be extremely important for the path integral approach to quantum gravity. Then instanton type configurations [22] near the classical ones, i.e. Einstein spaces, are more probable then the 'spurious'

1 E-mail: ekke@xanum.uam.mx

2 It has to be kept in mind that Newton's gravitational constant $G$ is one of the less precise known constants of physics. In order to improve this situation, there are plans [1] to measure the gravitational attraction of two bodies in a spaceship (Project SEE), where the larger body will function as a shepherd for the movement of the test mass, similarly as in the rings of Saturn. Amongst others, an accuracy of $3.3 \times 10^{-7}$ in the determination of $G$ should be feasible. 
Thomson spaces, as one would expect naively. For the modified duality with a breaking of scale invariance, the transition amplitude peaks at classical Einstein spaces only. Alternatively, in a four-dimensional Yang-Mills theory gauging the de Sitter group [37, 54], scale invariance gets spontaneously broken by a pseudo-Goldstone type 'radius vector' [62], odd under CP, in order to recover the Hilbert-Einstein action plus the Euler term.

From the work of Stelle [59] we know that the curvature squared gravity in Riemannian spacetime is perturbatively renormalizable but unfortunately plagued with physical ghost, i.e. negative residues in the graviton propagator [36]. This finding has diminished the initial interest $[19,20]$ in such models.

Much more promising and elegant is to start from a purely topological classical action, proportional to the gravitational Pointrjagin (or Euler) invariant and then quantize this model by nilpotent BRST transformations generated by $s$. Such a topological action is not only completely metric-free, but moreover conformally invariant $[69,34]$ and can provide a consistent Topological Quantum Field Theory (TQFT) as shown by Witten $[68,9]$. Lateron, it was realized by Baulieu and Singer [6] that for Yang-Mills theory Witten's action is a gauge-fixed version of the classical topological action through the standard BRST quantization procedure. Then the total Lagrangian consists of a d-exact part, as exemplified by the Pontrjagin invariant of the structure group, as well as an $s$-exact piece accounting for the chosen gauge fixing. Modulo an exact form, the full Lagrangian turns out to be BRST-invariant.

In the case of gravity one can obtain a rather realistic gravitational 'background' dynamics, if we complete the topological action by gauge constraints, enforcing the Lorentz condition on the linear connection and a double duality on the curvature. Then the resulting model depends on the metric of spacetime only via the $s$-exact term. This conforms with the general expectation that it is ultimately the process of quantization which necessarily induces a physical scale into a primordial topological model. According to Faddeev [18], quantization amounts to a stable deformation of the classical Poisson algebra with dimensional transmutation due to the physical dimension $[\hbar]=[p][q]$ of Planck's constant $\hbar$.

In this paper, we investigated the BRST quantization of topological gravity, cf. Ref. [69], following essentially Baulieu and Singer [6], cf. also Refs. [5, 2, 7]. Moreover, its classical limit, corresponding to the most probable, extremal 'trajectories' in the Feynman path integral, is analyzed. In the case of gravity, these are classical configurations with self- or anti-self dual curvature. In order to lift this 'vacuum degeneracy', a modified double duality constraint is considered which explicitly breaks scale invariance. For torsionless configurations, we can demonstrate that only Einstein's GR, consistently coupled to the symmetrized energy-momentum current of matter fields, surface as lowenergy (long range) effective theory, satisfying all macroscopic tests. The paper focusses

on the main physical features of the model, deferring details of the formalism to three appendices.

\section{WEYL-YANG THEORY OF GRAVITY}

In 1974 Yang [70] tentatively considered the possible replacement of Einstein's general relativity (GR) by an affine gauge theory with a Yang-Mills type action. In fact, 
curvature-squared Lagrangians had been considered before, first in 1919 by Weyl [65] with the emphasis on scale invariance, and then later by Stephenson [60], Higgs [27], as well as Kilmister and Newman [31].

When written in differential forms, the Stephenson-Kilmister-Yang (SKY) Lagrangian is given by

$$
L_{\mathrm{SKY}}=-\frac{1}{2} R^{\alpha \beta} \wedge^{*} R_{\alpha \beta}
$$

Our notation follows closely Cartan's exterior calculus, cf. Ref. [26] for details. Then Yang's vacuum equations

$$
D^{*} R_{\alpha \beta}=0
$$

together with

$$
\left.\left.E_{\alpha}=\frac{1}{2}\left(e_{\alpha}\right\rfloor R^{\mu v} \wedge^{*} R_{\mu v}-R^{\mu v} \wedge e_{\alpha}\right\rfloor^{*} R_{\mu v}\right)=0
$$

follow from varying the Lagrangian with respect to the connection one-form $\Gamma^{\alpha \beta}:=$ $\Gamma_{i}^{\alpha \beta} d x^{i}$ as well as with respect to the coframe $\vartheta^{\alpha}=e_{j}{ }^{\alpha} d x^{j}$.

The short-time initial value problem of Eg. (2) is well-posed [24]. Moreover, it does not depend on any length scale, i.e. it is scale invariant as envisioned by Weyl [66]. In this aspect it would be a good starting point for quantization, if one could avoid the physical ghost in the propagator of the gravitons. Consequently, a related purely topological action, in which the Hodge dual * in (1) is dismissed, could be more promising.

\section{TOPOLOGICAL ACTION}

Adopting ideas of Witten [68] for a topological Yang-Mills theory (TYM) and replacing the internal $S U(N)$ group by the linear group $S L(4, R)$ of the tangent space embracing the Lorentz group $S O(1,3)$ (or $S O(4)$ in Euclidean space with signature sig $=0$ ) as subgroup, we start from the gravitational Pontrjagin four-form

$$
\begin{aligned}
L_{\text {Pontr }} & :=d C_{\mathrm{RR}}=\operatorname{Tr}\{\Omega \wedge \Omega\} \\
& =\frac{1}{2} R_{\alpha}{ }^{\beta} \wedge R_{\beta}{ }^{\alpha}=\frac{1}{2} d\left(\Gamma_{\alpha}{ }^{\beta} \wedge R_{\beta}{ }^{\alpha}-\frac{1}{3} \Gamma_{\alpha}{ }^{\beta} \wedge \Gamma_{\beta}{ }^{\gamma} \wedge \Gamma_{\gamma}{ }^{\alpha}\right),
\end{aligned}
$$

which locally is a d-exact form violating parity $P$, our 'Clifform' notation is summarized in Appendix A following Ref. [42]. This Lagrangian is completely metric-free and as well invariant under the topological BRST transformations $s$ modulo an exact form, i.e.

$$
s L_{\mathrm{Pontr}}=2 d \operatorname{Tr}\{\Psi \wedge \Omega\}=d\left(\Psi_{\alpha}^{\beta} \wedge R_{\beta}{ }^{\alpha}\right) .
$$

Here $\Psi$ the one-form of the topological ghost with values in the Lie algebra of the linear group and the topological BRST transformations $s$ are specified in the Appendix B. A similar result would hold for the CP-invariant Euler term (28), i.e. $s L_{\text {Euler }}=$ $(-1)^{\text {sig }+1} 2 d\left(\Psi^{\alpha \beta} \wedge R_{\alpha \beta}^{(\star)}\right)$. However, the latter is only partial metric-free, since it involves the signature sig of the metric implicitly in the definition of the Lie dual, cf. Ref. [11]. 
This purely topological action can be amended by any $s$-exact form, provided $s$ is a nilpotent BRST transformation, i.e. one with $s^{2}=0$ without affecting the nice properties of the topological action. Following Baulieu and Singer [6], we may choose the Lorentz type conditions $d^{*} \Gamma=0, D^{*} \Psi=0$ on the connection and the topological ghost as well as the self-or anti-selfduality ${ }^{ \pm} \Omega^{( \pm)}=0$ of the curvature as gauge constraints consistently implemented via the Faddeev-Popov type Lagrangian

$$
\begin{aligned}
L_{\mathrm{FP}} & :=s \operatorname{Tr}\left\{\bar{\chi} \wedge{ }^{ \pm} \Omega^{( \pm)}+\bar{\Phi} D^{*} \Psi+\frac{1}{2} \rho \bar{\chi} \wedge b+\bar{c} d^{*} \Gamma+\frac{1}{2} \bar{c} \wedge{ }^{*} B\right\} \\
& =-\frac{1}{2} s\left[\bar{\chi}^{\alpha \beta} \wedge{ }^{ \pm} R_{\alpha \beta}^{( \pm)}+\bar{\Phi}_{\alpha \beta} D^{*} \Psi^{\alpha \beta}+\frac{1}{2} \rho \bar{\chi}^{\alpha \beta} \wedge b_{\alpha \beta}+\widetilde{\Psi}\right]
\end{aligned}
$$

where the trace is over the generators of the Lie algebra. Such a constraint, at the same time, constricts the linear group $S L(4, R)$ to the Lorentz (or $4 \mathrm{D}$ orthogonal) group as a subgroup. For comparison, also its component form is given. In order to end up with a four-form Lagrangian, the auxiliary fields $\bar{\chi}$ and $b$ have to be selfdual two-forms. In this framework, the so-called fermionic constraint is $\widetilde{\Psi}:=\bar{c}_{\alpha \beta} d^{*} \Gamma^{\alpha \beta}+\frac{1}{2} \bar{c} \wedge{ }^{*} B$.

In the following we are going to demonstrate that the full topological gravity Lagrangian

$$
L_{\mathrm{TG}}=d C_{\mathrm{RR}}+L_{\mathrm{FP}}
$$

is BRST invariant and classically equivalent to the self- or anti-selfdual version of SKY gravity as given by Eq. (1).

\section{EFFECTIVE SELFDUAL SKY GRAVITY}

Performing the BRST transformation $s$ in the gauge-fixing Lagrangian (6), we obtain after a long but straightforward calculation using the rules of Appendix B the result

$$
\begin{aligned}
L_{\mathrm{FP}}= & -\frac{1}{2} \operatorname{Tr}\left\{b \wedge{ }^{ \pm} \Omega^{( \pm)}+\bar{\chi} \wedge D \Psi-\bar{\chi} \wedge\left[c,{ }^{ \pm} \Omega^{( \pm)}\right]\right. \\
& +\bar{\eta} D^{*} \Psi+\bar{\Phi} D^{*} D \Psi+\bar{\Phi}\left[\Psi,{ }^{*} \Psi\right]+B d^{*} \Gamma \\
& \left.-\bar{c} d^{*} \Psi+\bar{c} d^{*}(D c)+\frac{1}{2} \rho b \wedge b+\frac{1}{2} B \wedge^{*} B\right\} .
\end{aligned}
$$

The variation with respect to the auxiliary field $B$ yields the Lorentz type condition $d^{*} \Gamma={ }^{*} B$ on the connection. Moreover, for vanishing real gauge parameter $\rho=0$, the equation of motion for the two-form $b$ enforces the self- or anti-self double duality condition

$$
{ }^{ \pm} \Omega^{( \pm)}:=\frac{1}{2}\left(\Omega \pm{ }^{*} \Omega^{(\star)}\right)=0
$$

on the curvature two-form $\Omega$, where we distinguish between the Hodge dual ${ }^{*}$ and the Lie dual $\left.{ }^{(}\right)$in a space(-time) of signature sig.

In the case of the choice $\rho=1$ of the real gauge parameter, the two-form $b$ is present in two terms, but then can be eliminated by a Gaussian integration in Euclidean space 
such that, up to gauge fixing terms, the SKY Lagrangian remains supplemented by the topological Euler term (28) as a boundary term, i.e.,

$$
\begin{aligned}
L_{\mathrm{SKY}}^{(\star)} & =\frac{1}{2} \operatorname{Tr}\left({ }^{ \pm} \Omega^{( \pm)} \wedge{ }^{ \pm} \Omega^{( \pm)}\right) \\
& =-\frac{1}{2} R^{\alpha \beta} \wedge{ }^{*} R_{\alpha \beta}-\frac{(-1)^{\mathrm{sig}}}{2} R^{\alpha \beta} \wedge R_{\alpha \beta}^{(\star)} \\
& =-\frac{1}{4}\left(R_{\alpha \beta}+{ }^{*} R_{\alpha \beta}^{(\star)}\right) \wedge{ }^{*}\left(R^{\alpha \beta}+{ }^{*} R^{\alpha \beta(\star)}\right) .
\end{aligned}
$$

The above argument is similarly to the path-integral approach to gravity, where the transition amplitude $\int \mathscr{D} \Gamma \exp \left[-\int V_{\mathrm{SKY}}^{(\star)} d^{4} x / \hbar\right]$ is evaluated in an imaginary 'spacetime' with Euclidean signature, cf. Ref. [40]. It is obvious from the equivalent binomial form of the effective SKY Lagrangian that anti-selfdual solutions [38, 8, 46] as well as the selfdual spaces

$$
R_{\alpha \beta}=\mp^{*} R_{\alpha \beta}^{(\star)}
$$

are extrema, but the minimum contributes the most to the transition amplitude. (For Euclidean signature sig $=1$, their solutions correspond to gravitational instantons, cf. Ref. [17].) Both constraints satisfy Yang's equation (2) due to the Bianchi identity (25) for the Lie dual of the curvature as well as the condition (3) of vanishing gauge field energy, typical for instantons. According to (29) the first case corresponds to Einstein spaces, annihilating the corresponding partially topological action, whereas the selfdual case induces Thompson spaces [61]. This 'vacuum degeneracy' was known already to Fairchild [19, 20] and discussed in more detail in Ref. [64], where the anti-selfdual SKY model has been coined "Yang-Mielke" theory of gravity.

Concentrating on topological terms such as those of Pontrjagin (26) and Euler (28), related self-dual modifications have also been advocated as topological $4 D$ selfdual gravity by Nakamichi et al. [49]. There, self- or anti-selfdual solutions are 'living' on Einstein spaces, as well. Moreover, deformations of conformal gravitational instantons can be classified topologically, cf. Ref. [55]. The addition of the Pontrjagin term with respect to the Riemannian curvature $R_{\alpha \beta}^{\{\}}$and the axial torsion one-form $\mathscr{A}:={ }^{*}\left(\vartheta_{\alpha} \wedge\right.$ $\left.T^{\alpha}\right)$ is rather well motivated by the axial anomaly

$$
\left\langle d j_{5}\right\rangle=2 i^{*} m\left\langle\bar{\psi} \gamma_{5} \psi\right\rangle-\frac{1}{48 \pi^{2}}\left(R_{\alpha \beta}^{\{\}} \wedge R^{\{\} \alpha \beta}+\frac{1}{2} d \mathscr{A} \wedge d \mathscr{A}\right)
$$

in the coupling to Dirac fields $\psi$, cf. Refs. $[32,44]$ and the literature therein.

\section{MODIFIED DOUBLE DUAL GAUGE FIXING}

In order to lift the vacuum degeneracy of selfdual SKY gravity, we may impose instead a modified gauge constraint via

$$
L_{\mathrm{FP}}(R)=-s \frac{1}{2} \bar{\chi}^{\alpha \beta} \wedge\left(H-\theta_{\mathrm{L}}^{\star} \Omega_{\alpha \beta}^{(\star)}-\frac{\theta_{\mathrm{T}}^{*}}{2 \ell^{2}} \sigma\right),
$$


involving the curvature excitation $H:=\frac{i}{4} H_{\alpha \beta} \sigma^{\alpha \beta}$ associated with a general quadratic curvature Lagrangian $L_{\mathrm{QPG}}$ in Poincaré gauge framework, where

$$
H_{\alpha \beta}:=-\partial L_{\mathrm{PG}} / \partial R^{\alpha \beta}=-*\left(\sum_{N=1}^{6} b_{(N)}{ }^{(N)} R_{\alpha \beta}\right)
$$

can be expanded into the irreducible curvature pieces. The propagating modes and particle content of such a model are known from the work of Sezgin and van Nieuwenhuizen [58], cf. Ref. [33]. In addition, the Hilbert-de Donder gauge condition $d^{*} \gamma=0$ and further ghost constraints need to be added to comply with the BRST algebra of the coframe $\gamma:=\vartheta^{\alpha} \gamma_{\alpha}$ indicated in Appendix C.

Then there arises the modified double duality ansatz

$$
H_{\alpha \beta}(* *)=\theta_{\mathrm{L}}^{\star} R_{\alpha \beta}^{(\star)}+\frac{\theta_{\mathrm{T}}^{*}}{2 \ell^{2}} \eta_{\alpha \beta}
$$

for the rotational field momenta $[39,41,71]$ as a gauge constraint. Here $\theta_{\mathrm{T}}^{*}$, and $\theta_{\mathrm{L}}^{\star}$ are dimensionless constants related to the individual coupling constants in the $\theta$-type boundary terms (27) and (28). (The instanton solutions of Yang's theory of gravity, classified [38] already 1981, are a special case of the ansatz (15) for the choice $\theta_{\mathrm{T}}^{*}=0$ and $\theta_{\mathrm{L}}^{\star}=\mp 1$.) Interesting enough, it can be regarded as field redefinition (FR) of the linear connection $\Gamma$ such that (15) is induced, see Ref. [43] for details ${ }^{3}$.

\section{EINSTEIN EQUATION WITH INDUCED COSMOLOGICAL CONSTANT AND AXIDILATON COUPLING}

In accordance with the classical field equations of affine gauge theory, in the torsionless case we are then left with Eq. (5.8.29) of Ref. [26], i.e.

$$
-E_{\alpha}=\frac{\theta_{\mathrm{T}}^{*}}{2} R^{\{\} \beta \gamma} \wedge \eta_{\alpha \beta \gamma}-\theta_{\mathrm{T}}^{*} \Lambda_{\theta} \eta_{\alpha}=\ell^{2} \sigma_{\alpha}
$$

where $G:=\frac{1}{2} R^{\beta \gamma} \wedge \eta_{\alpha \beta \gamma} \gamma^{\alpha}$ is the usual Einstein-Cartan (EC) current three-form which is dual to the usual Einstein tensor $G_{i j}:=R i c_{i j}^{\{\}}-\frac{1}{2} g_{i j}$. In the case of the real gauge parameter $\rho=1$, the two-form $b$ can again be eliminated by a Gaussian integration such that, up to gauge fixing terms, a generalization of the dual SKY Lagrangian similar to the quadratic expression (9.8) of Ref. [40] remains.

In both cases, our gauge constraints induce the classical Einstein equations

$$
G_{\alpha}-\Lambda_{\theta} \eta_{\alpha}=\frac{\ell^{2}}{\theta_{\mathrm{T}}^{*}} \sigma_{\alpha}
$$

\footnotetext{
${ }^{3}$ In a rather ad hoc fashion, such a FR was applied in Ref. [53] to Euler and Pontrjagin type terms. However, such deformations change the latter four-forms from being anymore d-exact terms, thus prevailing a topological interpretation.
} 
for the Riemannian background with the symmetric Belinfante-Rosenfeld three-form $\sigma_{\alpha}:=\Sigma_{\alpha}-D^{\{\}} \mu_{\alpha}$ as source and an effective gravitational coupling constant $\kappa_{\text {eff }}=$ $\ell^{2} / \theta_{\mathrm{T}}^{*}$.

Thus by modifying the gauge constraint, the double duality relation (15) surfaces which eliminates the 'vacuum ambiguity' for the exact solutions of SKY gravity, and only Einstein spaces remain as classical 'background'. Due to the explicit appearance of a length scale $\langle\varphi\rangle \propto 1 / \ell$ in the ansatz (15), it is suggestive to associate this with a (sponteneous) symmetry breaking of the scale or Weyl invariance of the original Lagrangian (14), for instance in a model [25] dynamically coupled to a dilaton field $\varphi$, cf. Ref. [16]. In a Riemann-Cartan-Weyl spacetime, generalizations of Einstein's equations with axial torsion as exemplified in Eq. (30) and a Weyl covector coupling can arise. When they are induced by an axion $a$ and dilation $\varphi$ as potentials, a cancelation of the axial torsion part in the chiral anomaly (12) can be achieved. Similarly as in strings, both may even combine into a single complex scalar, the axidilaton $\Phi=a+i f_{\varphi} \exp \left(-\varphi / f_{\varphi}\right)$, cf. Refs. $[57,47,29]$. Moreover, the torsion-induced quintaxion may simulate 'dark energy' (DE) with interesting repercussions on the cosmological evolution [48]. The anharmonic oscillating phases of expansion and acceleration could be related to the observed epochs in our Universe.

In general, an induced cosmological constant

$$
\Lambda_{\theta}=-\frac{3 \theta_{\mathrm{T}}^{*}}{2 \ell^{2}\left(\theta_{\mathrm{L}}^{\star}+b_{6}\right)}
$$

of microscopic origin [39] is unavoidable with an interesting (Anti-) de Sitter background, resembling the intriguing AdS/CFT correspondence. Moreover, there are strong indications from supernova observations [3] that the present epoch of the Universe is dominated by 'dark energy' in form of a tiny cosmological constant. Moreover, a coupling of the Euler or Gauss-Bonnet term to a hypothetical scalar field may generate [15] some of the cosmological dynamics responsible for the transition from matter dominance to the acceleration of the present epoch associated with DE.

Consequently, there is still a valid avenue to a consistent quantization based on a topological version of selfdual SKY gravity, departing, in a gauge covariant approach, from a $d$-exact topological term. Due to the nilpotency of the corresponding BRST charges [45], the $s$-exact term can easily account for the necessary gauge constraints such as (15) implying Einsteinian gravity for the classical 'background'. This, to some extent, provides an answer to the issue already raised 1963 by Feynman [21], whether Einstein's GR, in view of its force-free geometrical concepts, needs to be quantized at all or if curved spacetime can be left as an arena for quantized (topological) fields to play out. One would like to see generalizations of our approach to affine topological gauge models of gravity based on superconnections $[50,51]$ including the Higgs field.

\section{ACKNOWLEDGMENTS}

I would like to thank Alí A. Rincón Maggiolo, Eric S. Romero and Dmitri Vassiliev for valuable comments. Moreover, (E.W.M.) acknowledges the support of the SNI and thanks Noelia, Markus Gérard Erik, and Miryam Sophie Naomi for encouragement. 


\section{APPENDIX A: RIEMANN-CARTAN GEOMETRY IN CLIFFORD ALGEBRA-VALUED EXTERIOR FORMS}

The coframe $\vartheta^{\alpha}=e_{j}^{\alpha} d x^{j}$ of dimension [length] and the dimensionless connection one-form $\Gamma_{\alpha}^{\beta}=\Gamma_{i \alpha}{ }^{\beta} d x^{i}$ are the gauge potentials of nonlinearly realized local translations [62] and local linear transformations, respectively. The dual basis $\left\{\eta_{\alpha}, \eta_{\alpha \beta}, \eta_{\alpha \beta \gamma}, \eta_{\alpha \beta \gamma \delta}\right\}$ of exterior forms can be generated from the volume four-form $\eta=\eta_{\alpha \beta \gamma \delta} \vartheta^{\alpha} \wedge \vartheta^{\beta} \wedge \vartheta^{\gamma} \wedge \vartheta^{\delta} / 4$ ! by consecutive interior products 」 via $\left.\eta_{\alpha}:=e_{\alpha}\right\rfloor \eta={ }^{*} \vartheta_{\alpha}$, etc. On a four-dimensional manifold with metric index sig, the Hodge dual of $p$-forms is almost involutive, i.e. ${ }^{* *} \alpha=(-1)^{p(4-p)+\operatorname{sig}} \alpha$. For spacetimes where $\operatorname{sig}=1$ holds, it induces an almost complex structure, $\mathrm{cf}$. [13]. In four dimensions, the Hodge dual applied to two-forms is conformally invariant [4].

For a concise formulation of the BRST transformations it is instrumental to reexpress the Riemann-Cartan structure of spacetime in terms of Clifford algebravalued differential forms: In the familiar Pauli representation [10], the 16 matrices $\left\{\mathbf{1}_{4}, \gamma_{\alpha}, \sigma_{\alpha \beta}, \gamma_{5}, \gamma_{5} \gamma_{\alpha}\right\}$, where $\sigma_{\alpha \beta}:=\frac{i}{2}\left(\gamma_{\alpha} \gamma_{\beta}-\gamma_{\beta} \gamma_{\alpha}\right)$ are the Lorentz generators and $\gamma_{5}=-i \gamma_{\hat{0}} \gamma_{\hat{1}} \gamma_{\hat{2}} \gamma_{\hat{3}}$, constitute a basis of the Clifford algebra in four dimensions with the defining relation

$$
\gamma_{\alpha} \gamma_{\beta}+\gamma_{\beta} \gamma_{\alpha}=2 o_{\alpha \beta} \mathbf{1}_{4}
$$

They are normalized by $\operatorname{Tr}\left(\gamma_{\alpha} \gamma_{\beta}\right)=4 o_{\alpha \beta}$ and $\operatorname{Tr}\left(\sigma_{\alpha \beta} \sigma^{\gamma \delta}\right)=8 \delta_{[\alpha}^{\gamma} \delta_{\beta]}^{\delta}$, where $[\alpha \beta]=$ $\frac{1}{2}(\alpha \beta-\beta \alpha)$ denotes the antisymmetrization of indices.

In terms of the Clifford algebra-valued coframe and connection

$$
\gamma:=\gamma_{\alpha} \vartheta^{\alpha}, \quad \Gamma:=\frac{i}{4} \Gamma^{\alpha \beta} \sigma_{\alpha \beta}=\Gamma^{\{\}}-K,
$$

where $K:=\frac{i}{4} K^{\alpha \beta} \sigma_{\alpha \beta}$ is the contortion one-form, the $\overline{S O}_{\circ}(1,3) \cong S L(2, C)$-covariant exterior derivative $D=d+[\Gamma$,$] employs the algebra-valued form commutator$ $[\Psi, \Phi]:=\Psi \wedge \Phi-(-1)^{p_{1} p_{2}} \Phi \wedge \Psi$. The Hilbert-de Donder and Lorentz type gauge conditions involve the Hodge dual and can be rewritten as the following conditions on four-forms

$$
d^{*} \gamma=0, \quad d^{*} \Gamma=0 .
$$

Differentiation of these basic variables leads to the Clifford algebra-valued torsion and curvature two-forms

$$
\begin{aligned}
\Theta:=D \gamma & =T^{\alpha} \gamma_{\alpha}=\left(d \vartheta^{\alpha}+\Gamma_{\beta}{ }^{\alpha} \wedge \vartheta^{\beta}\right) \gamma_{\alpha}, \\
\Omega & :=d \Gamma+\Gamma \wedge \Gamma=\frac{i}{4} R^{\alpha \beta} \sigma_{\alpha \beta}=\frac{i}{4}\left(d \Gamma_{\alpha}{ }^{\beta}-\Gamma_{\alpha}{ }^{\gamma} \wedge \Gamma_{\gamma}{ }^{\beta}\right) \sigma^{\alpha}{ }_{\beta},
\end{aligned}
$$

respectively. The compact Clifform formula for the curvature was already known to Schrödinger [56]. In a RC spacetime, the translational and Lorentz-rotational ChernSimons terms read

$$
C_{\mathrm{TT}}:=\frac{1}{8 \ell^{2}} \operatorname{Tr}(\gamma \wedge \Theta)=\frac{1}{2 \ell^{2}} \vartheta^{\alpha} \wedge T_{\alpha}=-\frac{(-1)^{\mathrm{sig}}}{2 \ell^{2}} * \mathscr{A},
$$




$$
C_{\mathrm{RR}}:=-\operatorname{Tr}\left(\Gamma \wedge \Omega-\frac{1}{3} \Gamma \wedge \Gamma \wedge \Gamma\right),
$$

where $\mathscr{A}:={ }^{*}\left(\vartheta_{\alpha} \wedge T^{\alpha}\right)=\mathscr{A}_{i} d x^{i}$ is the axial torsion one-form. The translational ChernSimons term is not Weyl invariant, cf. (3.14.9) of Ref. [26], due to the occurence of a fundamental length $\ell$. (In view of (19), the Clifford algebra approach has the advantage in employing the trace in the definition (23), whereas the usual generators $P_{\alpha}$ of translations commute and do not have a non-degenerate Cartan-Killing metric.)

The Ricci identity for a p-form $\Psi$ reads

$$
D D \Psi=[\Omega, \Psi] .
$$

In RC geometry, the first and second Bianchi identity adopt the form

$$
D \Theta \equiv[\Omega, \gamma], \quad D \Omega \equiv 0, \quad D \Omega^{(\star)} \equiv 0,
$$

respectively, cf. Ref. [42] for further details. Here the Lie dual of the curvature is defined by $R_{\alpha \beta}^{(\star)}:=\frac{1}{2} \eta_{\alpha \beta \gamma \delta} R^{\gamma \delta}$.

The Lagrangians corresponding to the Bianchi identities (25) are the boundary terms

$$
\begin{aligned}
L_{\mathrm{NY}} & :=d C_{\mathrm{TT}}=\frac{1}{2 \ell^{2}}\left(T^{\alpha} \wedge T_{\alpha}+R_{\alpha \beta} \wedge \vartheta^{\alpha} \wedge \vartheta^{\beta}\right) \\
L_{\mathrm{Pontr}} & :=d C_{\mathrm{RR}}=\frac{1}{2} R_{\alpha}^{\beta} \wedge R_{\beta}^{\alpha} \\
& =-\frac{1}{2} R_{\alpha \beta}^{\{\}} \wedge R^{\{\} \alpha \beta}-\frac{1}{12} d\left[* \mathscr{A} \wedge R^{\{\}}-\frac{1}{3} \mathscr{A} \wedge d \mathscr{A}+\frac{1}{9} * \mathscr{A} \wedge^{*}(\mathscr{A} \wedge * \mathscr{A})\right](27)
\end{aligned}
$$

The latter contains, amongst others, a term proportional to the Riemannian curvature scalar $R^{\{\}}:={ }^{*}\left(R^{\{\} \alpha \beta} \wedge \eta_{\beta \alpha}\right)$ and the axial torsion piece $d \mathscr{A} \wedge d \mathscr{A}$ of the axial anomaly $[32,43]$ with a relative factor 9 . Up to normalizations, the four-forms (26) and (27) are known as Nieh-Yan [52] and gravitational Pontrjagin term, respectively.

On the other hand, the topological Euler term

$$
\begin{aligned}
L_{\text {Euler }} & :=(-1)^{\operatorname{sig}+1} \operatorname{Tr}\left\{\Omega \wedge \Omega^{(\star)}\right\}=\frac{(-1)^{\text {sig }}}{2} R^{\alpha \beta} \wedge R_{\alpha \beta}^{(\star)} \\
& =\frac{(-1)^{\mathrm{sig}}}{2} d\left(\Gamma_{\alpha \beta} \wedge R^{\alpha \beta(\star)}-\frac{1}{3} \Gamma_{\alpha}^{\beta(\star)} \wedge \Gamma_{\beta}^{\gamma} \wedge \Gamma_{\gamma}^{\alpha}\right) \\
& \equiv \frac{1}{2} R_{\alpha \beta} \wedge^{*} R^{\alpha \beta}-2 \operatorname{Ric}_{\alpha \beta} \wedge^{*} \operatorname{Ric}^{\alpha \beta}+\frac{1}{2} \operatorname{Ric}_{\alpha}{ }^{\alpha} \wedge^{*} \operatorname{Ric}_{\beta} \beta
\end{aligned}
$$

for Riemann-Cartan spaces has an equivalent representation in terms of Yang's Lagrangian $V_{\mathrm{SKY}}$ as well as a Ricci-squared and a curvature scalar squared term, cf. Eq. (3.1) of Ref. [38]. The expression in terms of the symmetric Ricci tensor, i.e. the zeroform $\operatorname{Ric}_{\alpha \beta}:=(-1)^{\mathrm{sig} *}\left(R_{(\alpha}{ }^{\delta} \wedge \eta_{\delta \mid \beta)}\right)$, is also known as Gauss-Bonnet term.

Due to the algebraic Lanczos identity [35], the double dual curvature

$$
\left.{ }^{*} R_{\alpha \beta}^{(\star)} \equiv(-1)^{\operatorname{sig}} R_{\alpha \beta}+e_{[\alpha}\right\rfloor G_{\beta]}+\frac{1}{4} R \eta_{\alpha \beta}+D_{[\alpha} T_{\beta]}
$$


can be written in terms of the contraction of the EC three-form $G_{\alpha}:=R^{\beta \gamma} \wedge \eta_{\alpha \beta \gamma} / 2$ and the curvature scalar $\left.\left.R:=e_{\beta}\right\rfloor e_{\alpha}\right\rfloor R^{\alpha \beta}$, a zero form; cf. Ref. [38] for the same result in Riemannian spacetime and in components.

Likewise, the Einstein-Cartan three-form

$$
\begin{aligned}
G & :=\frac{1}{2} R^{\beta \gamma} \wedge \eta_{\alpha \beta \gamma} \gamma^{\alpha} \\
& \left.\left.=G^{\{\}}+\frac{(-1)^{\mathrm{sig}}}{12}\left(e_{\alpha}\right\rfloor \mathscr{A} \wedge^{*} \mathscr{A}-\frac{1}{3} \mathscr{A} \wedge e_{\alpha}\right\rfloor^{*} \mathscr{A}\right) \gamma^{\alpha}+\frac{(-1)^{\mathrm{sig}}}{6} \gamma \wedge d \mathscr{A} \text { (30) }
\end{aligned}
$$

decomposes into the Einstein three-form $G^{\{\}}=G_{\alpha}{ }^{\beta} \eta_{\beta} \gamma^{\alpha}$ with respect to the Riemannian connection $\Gamma^{\{\}}$and axial torsion pieces, see Ref. [48] for details.

\section{APPENDIX B: BRST TRANSFORMATIONS}

In the BRST formalism, the infinitesimal gauge transformations are converted, via ghosts, into operator transformations. Let $c:=\frac{i}{4} c^{\alpha \beta} \sigma_{\alpha \beta}$ denote the zero-form of the usual Faddeev-Popov ghost, cf. Refs. [45, 63], $\Psi:=\frac{i}{4} \Psi_{j}^{\alpha \beta} \sigma_{\alpha \beta} d x^{j}$ the topological ghost one-form and $\Phi:=\frac{i}{4} \Phi^{\alpha \beta} \sigma_{\alpha \beta}$ the corresponding ghost of the topological ghost. All are Lie algebra-valued due to the appearance of the generator $\sigma_{\alpha \beta}$ of the linear (or Lorentz) group.

Then the global BRST transformations generated by the zero-form $s$ take the form

$$
\begin{aligned}
s \Gamma & =\Psi-D c, \\
s c & =\Phi-\frac{1}{2}[c, c], \\
s \Omega & =D \Psi-[c, \Omega], \\
s \Psi & =-D \Phi-[c, \Psi], \\
s \Phi & =-[c, \Phi] .
\end{aligned}
$$

This is consistent with the interpretation of $\Gamma$ and $\Omega$ as connection one-form and gauge two-form, respectively. The topological ghost $\Psi$ complements the inhomogeneous transformation law of infinitesimal gauge fields. By construction, these BRST transformations are nilpotent for all variables, i.e. $s^{2}=0$.

In the rather elegant geometrical interpretation of Ref. [6], the graded $^{4}$ connection and curvature defined via

$$
\widetilde{\Gamma}:=\Gamma \oplus c, \quad \widetilde{\Omega}:=\Omega \oplus \Psi \oplus \Phi,
$$

\footnotetext{
4 The grading permits using the direct sum $\oplus$ of exterior forms carrying different form degree $p$ and ghost number $g$, such that the graded commutator is now defined by $[\Psi, \Phi]:=\Psi \wedge \Phi-(-1)^{\left(p_{1}+g_{1}\right)\left(p_{2}+g_{2}\right)} \Phi \wedge \Psi$, cf. Ref. [14].
} 
satisfy the corresponding graded structure equation and second Bianchi identity for the graded curvature, i.e.

$$
\begin{gathered}
(d \oplus s) \widetilde{\Gamma}+\frac{1}{2}[\widetilde{\Gamma}, \widetilde{\Gamma}]=\widetilde{\Omega} \\
(d \oplus s) \widetilde{\Omega}+[\widetilde{\Gamma}, \widetilde{\Omega}] \equiv 0
\end{gathered}
$$

They comprise all the BRST transformations (31) above, and constitute an ordinary de Rham cohomology. Moreover, a straightforward proof of the nilpotency of the BRST operator $s$ now follows simply from $(d \oplus s)(d \oplus s) \equiv 0$ as a result of the graded Bianchi identity (33), the anti-commutation of the graded commutator $[s, d]=0$, and the Poincaré lemma $d d \equiv 0$.

In order to implement the gauge constraints one uses the antifield formalism, where the Lorentz algebra-valued antighosts $\bar{c}, \bar{\chi}$, and $\bar{\Phi}$ obey the following BRST transformation rules

$$
\begin{aligned}
s \bar{c}=B, & & s B=0, \\
s \bar{\chi}=b, & & s b=0, \\
s \bar{\Phi}=\bar{\eta}, & & s \bar{\eta}=0 .
\end{aligned}
$$

By construction $s$ is nilpotent for the antighosts, since the Lagrangian multiplier $\bar{\eta}$, the selfdual two-forms $B$ and $b$ are auxiliar fields introduced as trivial pairs. A symmetric ghost/antighosts spectrum of an extended BRST invariance could be obtained via a field redefinition [12]. (For metric-affine gravity, an antifield formalism has been developed [23] without employing topological ghosts.)

By introducing a BRST gauge field $\alpha=\alpha_{i} d x^{i}$ with ghost number -1 and a commuting ghost $\lambda$ of $\alpha$, one can promote [2] the global BRST transformations (31) into a local ones, where

$$
s_{1}(\alpha+\lambda)=-d \lambda, \quad s_{1} \lambda=0
$$

is satisfying the algebra

$$
\left(d \oplus s_{1}\right)(\alpha+\lambda)=d \alpha .
$$

The cohomology (33) of the BRST transformation remains unchanged by this promotion which, likewise, can be generated via the field redefinitions $c \rightarrow(\alpha+\lambda) c, \Psi \rightarrow(\alpha+$ $\lambda) \Psi$ as well as $\Phi \rightarrow(\alpha+\lambda)^{2} \Phi$ of the ghosts, see Ref. [2] for more details. Thus, local BRST invariance of an action puts no more restrictions on its form than the usual global one: The gauge field $\alpha$ is only present to compensate for the enlargement of the symmetry, from global to local, but it cannot propagated, due to its non-vanishing ghost number.

\section{APPENDIX C: BRST TRANSLATIONS AND DIFFEOMORPHISMS}

By gauging the translational part $R^{4}$ of the affine group [26], there arises a translational connection $\Gamma^{(\mathrm{T})}$ which in gravity is usually 'soldered' [62] to the base manifold, with the familiar tetrads or coframe $\gamma$ as the result, cf. (20). Then the topological structure 
equations (33) for the linear connection get amended by the corresponding graded first Cartan structure equation and the first Bianchi identity

$$
\begin{aligned}
(d \oplus s) \gamma+[\widetilde{\Gamma}, \gamma] & =\widetilde{\Theta} \\
(d \oplus s) \widetilde{\Theta}+[\widetilde{\Gamma}, \widetilde{\Theta}] & \equiv[\widetilde{\Omega}, \gamma]
\end{aligned}
$$

respectively, for the graded torsion two-form $\widetilde{\Theta}:=\Theta \oplus \psi \oplus \phi$. (When undoing the 'soldering', the translation connection $\Gamma^{(\mathrm{T})}$ would need to be graded as well, e.g. by the substitution $\gamma \rightarrow \widetilde{\gamma}:=\gamma \oplus c$ in the first structure equation.)

In topological models of gravity, diffeomorphisms can also be taken account of by generalizing via $s \rightarrow \widetilde{s}=s+\mathrm{七}_{\xi}$ the BRST transformations $s$ involving the covariant Lie derivative $\left.\left.\iota_{\xi}:=\xi\right\rfloor D-D \xi\right\rfloor$ built from the interior product $\rfloor$ with respect to an anticommuting ghost vector field $\xi=\xi^{i} \partial_{i}$. Note that there is sign difference in the definition of $\mathrm{Ł}_{\xi}$, since $\xi$ has ghost number one. Then the BRST algebra remains intact [7, 14], up to a redefinition of all ghosts by means of a similarity transformation generated by the formal exponential

$$
\left.\left.\left.\left.\left.\exp (\xi\rfloor):=\mathbf{1}+\xi\rfloor+\frac{1}{2 !} \xi\right\rfloor \xi\right\rfloor+\frac{1}{3 !} \xi\right\rfloor \xi\right\rfloor \xi\right\rfloor+\cdots
$$

In effect, the graded curvature and torsion in the cohomologies (33) and (37) are replaced by $\exp (\xi\rfloor) \widetilde{\Omega}$ and $\exp (\xi\rfloor) \widetilde{\Theta}$, respectively.

\section{REFERENCES}

1. A. D. Alexeev, K. A. Bronnikov, N. I. Kolosnitsyn, M. Y. Konstantinov, V. N. Melnikov and A. J. Sanders, "Measurement of the gravitational constant G in space (Project SEE): Sensitivity to orbital parameters and space charge effect," arXiv:gr-qc/0104066.

2. C. Aragao de Carvalho and L. Baulieu: "Local BRST symmetry and superfield formulation of the Donaldson-Witten theory," Phys. Lett. B 275, 323 (1992).

3. P. Astier et al., "The Supernova Legacy Survey: Measurement of $\Omega_{\mathrm{M}}, \Omega_{\Lambda}$ and $w$ from the first year data set," Astronomy and Astrophysics 447, 31 (2006).

4. M.F. Atiyah, N.J. Hitchin, and I.M. Singer, Proc. R. Soc. (London) A 362, 425 (1978).

5. L. Baulieu: "Perturbative gauge theories," Phys. Rept. 129, 1 (1985).

6. $\quad$ L. Baulieu and I. M. Singer: "Topological Yang-Mills symmetry,” Nucl. Phys. Proc. Suppl. 5B, 12 (1988).

7. L. Baulieu and A. Tanzini: “Topological gravity versus supergravity on manifolds with special holonomy," JHEP 0203, 015 (2002).

8. I. M. Benn, T. Dereli and R. W. Tucker, "Double dual solutions of generalized theories of gravitation," Gen. Rel. Grav. 13, 581 (1981).

9. D. Birmingham, M. Blau, M. Rakowski and G. Thompson: “Topological field theory,” Phys. Rept. 209, 129 (1991).

10. J.D. Bjorken and S.D. Drell: Relativistic Quantum Mechanics (Mc Graw-Hill, New York 1964).

11. M. Blau and G. Thompson: "Do metric independent classical actions lead to topological field theories?," Phys. Lett. B 255, 535 (1991).

12. N. R. F. Braga and C. F. L. Godinho: "Extended BRST invariance in topological Yang-Mills theory revisited," Phys. Rev. D 61, 125019 (2000). 
13. C.H. Brans, J. Math. Phys. 15, 1559 (1974); 16, 1008 (1975).

14. L. N. Chang and C. P. Soo: "BRST cohomology and invariants of four-dimensional gravity in Ashtekar variables," Phys. Rev. D 46, 4257 (1992).

15. G. Cognola, E. Elizalde, S. Nojiri, S. Odintsov and S. Zerbini, "String-inspired Gauss-Bonnet gravity reconstructed from the universe expansion history and yielding the transition from matter dominance to dark energy," Phys. Rev. D 75, 086002 (2007).

16. T. Dereli and R. W. Tucker: "A broken gauge approach to gravitational mass and charge," JHEP 0203, 041 (2002).

17. T. Eguchi, P. B. Gilkey and A. J. Hanson: "Gravitation, gauge theories and differential geometry," Phys. Rept. 66, 213 (1980).

18. L.D. Faddeev: "How we understand "quantization" a hundred years after Max Planck", Phys. Bl. 52, 689 (1996).

19. E. E. Fairchild: "Gauge theory of gravitation," Phys. Rev. D 14, 384 (1976).

20. E. E. Fairchild: "Yang-Mills formulation of gravitational dynamics," Phys. Rev. D 16, 2438 (1977).

21. R. P. Feynman, F. B. Morinigo, W. G. Wagner and B. Hatfield (eds): "Feynman lectures on gravitation," (Addison-Wesley, Reading, USA 1995) 232 p.

22. C. H. Gu, H. S. Hu, D. Q. Li, C. L. Shen, Y. L. Xin and C. N. Yang: "Riemannian spaces with local duality and gravitational instantons," Sci. Sin. 21, 475 (1978).

23. F. Gronwald: "BRST-antifield treatment of metric-affine gravity," Phys. Rev. D 57, 961 (1998).

24. B. S. Guilfoyle and B. C. Nolan: "Yang's gravitational theory," Gen. Rel. Grav. 30, 473 (1998).

25. F.W. Hehl, J.D. McCrea, E.W. Mielke, and Y. Ne'eman: "Progress in metric-affine gauge theories of gravity with local scale invariance", Found. Phys. 19, 1075 - 1100 (1989).

26. F.W. Hehl, J.D. McCrea, E.W. Mielke, and Y. Ne'eman, Phys. Rept. 258 (1995) 1- 171.

27. P.W. Higgs, Nuovo Cimento 11, 816 (1959).

28. D. J. Kapner, T. S. Cook, E. G. Adelberger, J. H. Gundlach, B. R. Heckel, C. D. Hoyle and H. E. Swanson, "Tests of the gravitational inverse-square law below the dark-energy length scale," Phys. Rev. Lett. 98, 021101 (2007).

29. R. K. Kaul: "Gauge theory of gravity and supergravity," Phys. Rev. D 73, 065027 (2006).

30. T.W.B. Kibble and K.S. Stelle: "Gauge theories of gravity and supergravity", in Progress in Quantum Field Theory, Festschrift for Umezawa, H. Ezawa and S. Kamefuchi, eds. (Elsevier Science Publ. Amsterdam 1986), p. 57.

31. C.W. Kilmister and D.L. Newman, Proc. Cambridge Phil. Soc. (Math. Phys. Sci.) 57, 851 (1961).

32. D. Kreimer and E.W. Mielke: "Comment on: Topological invariants, instantons, and the chiral anomaly on spaces with torsion", Phys. Rev. D63 048501 (2001).

33. R. Kuhfuß and J. Nitsch, Gen. Rel. Grav. 18, 1207 (1986).

34. J. M. F. Labastida and M. Pernici, "A Lagrangian for topological gravity and its BRST quantization," Phys. Lett. B 213, 319 (1988).

35. C. Lanczos: "A remarkable property of the Riemann-Christoffel tensor in four dimensions", Ann. Math. 39, 842 (1938).

36. C. Y. Lee and Y. Ne'eman: "Renormalization of gauge affine gravity," Phys. Lett. B 242, 59 (1990).

37. S. W. MacDowell and F. Mansouri: "Unified geometric theory of gravity and supergravity," Phys. Rev. Lett. 38, 739 (1977) [Erratum-ibid. 38, 1376 (1977)].

38. E.W. Mielke: "On pseudoparticle solutions in Yang's theory of gravity", Gen. Rel. Grav. 13 (1981) $175-187$.

39. E. W. Mielke, J. Math. Phys. 25, 663 (1984).

40. E. W. Mielke: "On pseudoparticle solutions in the Poincaré gauge theory of gravity", Fortschr. Phys. 32, 639 (1984).

41. E.W. Mielke: "Ashtekar's complex variables in general relativity and its teleparallelism equivalent", Ann. Phys. (N.Y.) 219, 78- 108 (1992)

42. E. W. Mielke: "Beautiful gauge field equations in Clifforms", Int. J. Theor. Phys. 40 (2001) 171 190.

43. E.W. Mielke: "Duality and renormalization scheme for Einsteinian gravity as a fix point within a gravitational gauge framework", Electronic Journal of Theoretical Physics (EJTP) 3, No. 12, 1-18 (2006).

44. E.W. Mielke: "Anomalies and gravity", in: Particles and Fields, Commemorative Volume of the Division of Particles and Fields of the Mexican Phys. Soc., Morelia Michoacán, 6-12 Nov. 2005, 
Part B., M.A. Pérez, L.F. Urrutia, and L. Villaseñor, eds. (AIP Conference Proc., Melville N.Y. 2006) Vol. 857, pp. $246-257$.

45. E.W. Mielke and A. A. Rincón Maggiolo: "Algebra for a BRST quantization of metric-affine gravity", Gen. Rel. Grav. 35, 771-789 (2003).

46. E.W. Mielke and A. A. Rincón Maggiolo: "Duality in Yang's theory of gravity", Gen. Rel. Grav. 37, 997-1007 (2005).

47. E. W. Mielke and F. E. Schunck, “Are axidilaton stars massive compact halo objects?," Gen. Rel. Grav. 33, 805 (2001).

48. E. W. Mielke and E. S. Romero: "Cosmological evolution of a torsion-induced quintaxion," Phys. Rev. D 73, 043521 (2006).

49. A. Nakamichi, A. Sugamoto, and I. Oda, Phys. Rev. D 44 (1991) 3835.

50. Y. Ne'eman: "A superconnection for Riemannian gravity as spontaneously broken SL $(4, \mathrm{R})$ gauge theory," Phys. Lett. B 427, 19 (1998).

51. Y. Ne'eman, S. Sternberg and D. Fairlie, "Superconnections for electroweak su(2/1) and extensions, and the mass of the Higgs," Phys. Rept. 406, 303 (2005).

52. H.T. Nieh and M.L. Yan, J. Math. Phys. 23 (1982) 373-374.

53. Y. N. Obukhov and F. W. Hehl, "On the relation between quadratic and linear curvature Lagrangians in Poincaré gauge gravity," Acta Phys. Polon. B 27, 2685 (1996).

54. H. R. Pagels: "Gravitational gauge fields and the cosmological constant," Phys. Rev. D 29, 1690 (1984).

55. M. J. Perry and E. Teo, “Topological conformal gravity in four-dimensions," Nucl. Phys. B 401, 206 (1993).

56. E. Schrödinger: "Diracsches Elektron im Schwerefeld I.", Sitzungsber. Preuss. Akad. Wiss. Phys. Math. Kl. 11, 105 (1932).

57. A. Sen, Phys. Lett. A 8, 2023 (1993).

58. E. Sezgin and P. van Nieuwenhuizen, Phys. Rev. D21 (1980) 3269-3280.

59. K.S. Stelle, Phys. Rev. D16 (1977) 953.

60. G. Stephenson, Nuovo Cimento 9 (1958) 263.

61. A.H. Thompson, Phys. Rev. Lett. 34, 505; 35, 320 (1975).

62. R. Tresguerres and E.W. Mielke: "Gravitational Goldstone fields from affine gauge theory", Phys. Rev. D62 44004 (2000).

63. J. W. Van Holten, “Aspects of BRST quantization,” Lect. Notes Phys. 659, 99 (2005).

64. D. Vassiliev: "Pseudoinstantons in metric-affine field theory", Gen. Rel. Grav. 34, 1239 (2002).

65. H. Weyl: "Eine neue Erweiterung der Relativitätstheorie", Ann. Phys. (Leipzig) IV. Folge, 59, 103 (1919).

66. H. Weyl: "Gravitation and the electron", Proc. Nat. Acad. Sci. (Washington) 15, 323 (1929).

67. C. M. Will, "Was Einstein right? Testing relativity at the centenary," Annalen Phys. 15, 19 (2005).

68. E. Witten:“Topological quantum field theory,” Commun. Math. Phys. 117, 353 (1988); 118, 411 (1988).

69. E. Witten:“Topological gravity,” Phys. Lett. B 206, 601 (1988).

70. C. N. Yang: "Integral formalism for gauge fields", Phys. Rev. Lett. 33, 445-447 (1974).

71. V.V. Zhytnikov, J. Math. Phys. 35 6001-6017 (1994). 